\title{
Dificuldades com Inferências Inválidas entre Adultos: Tentativa de Superação
}

\author{
Maria da Graça Bompastor Borges Dias ${ }^{12}$
}

Universidade Federal de Pernambuco

\begin{abstract}
Resumo
Estudos entre crianças e adultos demonstram que problemas silogísticos válidos (Modus Ponens e Modus Tollens) são de fácil solução, ao contrário daqueles que envolvem formas inválidas (Afirmação do Conseqüente e Negação do Antecedente), nos quais o desempenho dos sujeitos é muito baixo. No presente estudo, tentou-se minimizar o grande número de erros que ocorre nos silogismos inválidos utilizando inclusão de outra alternativa de resposta e contra-exemplo. Sujeitos universitários conseguiram grande número de acertos nessas formas, no entanto, o desempenho decaiu nas formas válidas. Já os adultos com pouca ou nenhuma escolarização parecem não ter sido influenciados pelas variáveis introduzidas, obtendo um melhor nível de desempenho nas formas válidas do que nas inválidas, como ocorreu em outros estudos.

Palavras-chave: Silogismos; formas válidas e inválidas; conversão inválida.
\end{abstract}

Difficulty with Invalid Inferences among Adults: Tentative of Superation

Abstract

Studies among children and adults showed that valid syllogistic problems (Modus Tollens and Modus Ponens) are easier to solve than invalid ones (Affirmation of Consequent and Negation of Antecedent) where subjects' performance is very low. In this study we tried to diminish the great number of errors in the invalid problems using another alternative for the responses and counter-example. University subjects obtained a great number of correct responses in the invalid forms, however their performance was low in the valid forms. The adults with low education and the illiterates did not seem to be influenced by the control variables introduced, showing a better level of performance in the valid forms then in the invalid ones, as others studies had shown.

Keywords: Syllogisms; valid and invalid form; invalid conversion.

Uma das formas de estudar o raciocínio lógico dos indivíduos tem sido analisar como eles tiram conclusões de premissas ou afirmativas. Segundo Falmagne (1975), a pessoa demonstraria estar raciocinando logicamente quando suas conclusões são tiradas com base apenas nas premissas oferecidas, sem considerar o conhecimento que tem do mundo ou aspectos externos ao conteúdo dado. Um silogismo é um tipo de argumento dedutivo que, segundo Mayer (1977), consiste de sentenças (duas premissas e uma conclusão), sendo cada uma das sentenças um dos quatro tipos de proposição categórica. Por exemplo:

Todos os homens são mortais.

Sócrates é um homem.

Portanto, Sócrates é mortal.

A primeira premissa é uma Afirmativa Universal, a segunda uma Afirmativa Particular, e uma conclusão também Particular.

Endereço para correspondência: Av. Beira Mar, 520, 81, Piedade, Jaboatão, PE, 54310-064. Fone: (81) 271 8272; Fax: 271 1843. E-mail: Mdias@npd.ufpe.br

2 Apoio CNPq e FACEPE
Existem quatro formas básicas de silogismos ou regras de inferências: Modus Ponens, Modus Tollens, Afirmação do Conseqüente e Negação do Antecedente. Os dois primeiros argumentos têm conclusões que são certas e logicamente necessárias: Modus Ponens ( $p$ implica $q, p$, portanto $q$ ) e Modus Tollens ( $p$ implica $q$, não $q$, portanto não $p$ ) e são chamados de inferências válidas.

Os outros dois argumentos, Afirmação do Conseqüente ( $p$ implica $q, q$, portanto...?) e Negação do Antecedente ( $p$ implica $q$, não $p$, portanto...?), possuem conclusões que são indeterminadas. Assim, "não posso dizer" é a resposta esperada nestas duas formas que são por isto chamadas de inválidas. Com estes e outros diferentes tipos de problemas silogísticos em mãos, estudiosos constataram que o raciocínio das pessoas é influenciado em grande escala por fatores tais como: forma dos silogismos, conteúdo das premissas e nível de escolarização.

\section{Forma dos Silogismos}

Estudos com crianças e com adultos têm demonstrado que problemas sob a forma válida Modus Ponens não apresentam dificuldades em serem 
solucionados (Dias, 1987; Dias \& Harris, 1988a; 1988b 1990; Rips \& Marcus, 1977; Shapiro \& O’Brien, 1970; Taplin \& Staudenmayer, 1973). No entanto, muitos desses estudos mostram que o bom desempenho dos sujeitos cai um pouco na outra forma válida (Modus Tollens) e é assustadoramente baixo nas formas inválidas (Afirmação do Conseqüente e Negação do Antecedente).

A razão de tão baixa performance nas formas inválidas foi denominada por Chapman e Chapman (1959) e Mayer (1977) de "conversão inválida". Nelas, os sujeitos tentam tirar conclusões (certeza ou falsidade) interpretando as premissas de modo bicondicional. Assim, transformam "Se A é verdadeiro então B é verdadeiro" em "Se B é verdadeiro então A é verdadeiro". Esforços têm sido feitos a fim de evitar essa conversão inválida.

Rumain, Connell e Braine (1983) conseguiram que, tanto adultos quanto crianças de sete a dez anos, melhorassem o desempenho nas formas inválidas expandindo a premissa maior, como por exemplo:

Se tem um pato na caixa,

então tem uma pêra na caixa (Simples)

Se tem um porco na caixa,

então tem uma maçã na caixa.

Se tem um cachorro na caixa

então tem uma laranja na caixa.

Se tem um tigre na caixa,

então tem uma laranja na caixa. (Complexa)

No entanto, juntamente com este tipo de expansão, chamada também pelos autores de "premissa complexa", outro tipo de variável foi apresentada aos sujeitos: uma caixa contendo uma fruta ou animal era mostrada para cada problema e, foi adicionada ainda a opção de resposta "Sem pistas suficientes" às outras duas opções "Sim e Não". Fica então a dúvida de qual das três variáveis (ou a interação das mesmas) seria mais efetiva aos resultados encontrados. Isto é, a introdução da premissa expandida o material (caixa com fruta ou animal) e a terceira opção de resposta "sem pistas suficientes". A inclusão de três alternativas de resposta, já havia sido verificada por Revlis (1975a), o qual encontrou com adultos que seus erros caiam em $50 \%$ nas formas inválidas.

Quanto a estudos com crianças, Dias e Ruiz (1990), realizaram uma investigação com idades de cinco e oito anos, na qual foi adicionado à premissa maior apenas uma frase que exemplificava outro fato pertencente a uma mesma categoria. Por exemplo:

Se é neve então é branco. (Simples)

Se é neve então é branco, mas algodão é branco também (Expandida)

Nos problemas inválidos, quando a condição "expandida" era apresentada, apesar de ter havido uma melhora significativa no desempenho das crianças, o número de erros foi ainda mais freqüente nas formas inválidas do que nas válidas. Evidencia-se então, que um modo realmente eficaz de bloquear a conversão inválida, parece não ter sido ainda encontrado nem para crianças nem para adultos.

Assim, no presente estudo, tenta-se minimizar a ocorrência de tantos erros nas formas inválidas de Afirmação do Conseqüente e Negação do Antecedente entre adultos com diferentes níveis de escolarização. A escolha desses tipos de participantes deve-se, dentre outras razões que serão apresentadas mais adiante, as evidências encontradas dentro da Teoria da Lógica Mental (ver Discussão) que apontam que existem esquemas básicos que dão conta das inferências que são feitas no dia-a-dia até por crianças pré-escolares (Modus Ponens e Modus Tollens) e outros esquemas, aplicáveis nas inferências inválidas (Afirmativas do Conseqüente e Negação do Antecedente), os quais o grau de escolarização parece influenciar o bom desempenho.

Como foi visto anteriormente, estudos como os de Dias e Ruiz (1990), Revlis (1975b); Rips e Marcus (1977) e Rumain, Connell e Braine (1983) tinham como objetivo buscar um bom nível e desempenho de crianças e adultos encontrado nas formas válidas, fosse alcançado nas formas inválidas; tais estudos obtiveram alguns resultados satisfatórios. No estudo de Revlis (1975a) com adultos, a informação dada aos sujeitos de que a "conclusão nãoválida" poderia ser uma das alternativas de resposta, diminuiu o número de erros. No entanto, em estudo similar, Rips e Marcus (1977) verificaram que apenas a apresentação dessa alternativa, não foi suficiente para aumentar o desempenho dos sujeitos nas inferências inválidas.

No estudo de Dias e Ruiz (1990), apesar de ter ocorrido uma diminuição da conversão inválida quando se expandia a premissa maior, as crianças ainda tendiam a responder positivamente à falácia de Afirmação do Conseqüente e negativamente à falácia de Negação do Antecedente, como o faziam corretamente às formas válidas Modus Ponens e Modus Tollens. Vale salientar que neste estudo não havia alternativas de respostas e todas as crianças utilizaram apenas respostas afirmativas ou negativas.

Como já foi relatado, adultos também denotam baixa performance nas formas inválidas (Revlis, 1975a; Rips \& Marcus, 1977; Wildman \& Fletcher, 1977). Em nossas observações em sala de aula de pós-graduação, verificamos que, ao explicar às inferências inválidas, a verbalização de que os alunos devem levar em consideração que "nem sempre a recíproca é verdadeira", 
tem eliciado uma diminuição no número de falácias. Este tipo de verbalização juntamente com a alternativa de resposta "pode ser e pode não ser" foram apresentados aos sujeitos. Neste estudo foi incluído um grupo de adultos analfabetos, outro com pouca escolarização e outro de estudantes universitários. Isto devido ao fato de que, em um estudo de Dias (1987), com universitários, analfabetos e sujeitos com pouca escolarização, não se tentou minimizar a conversão inválida mas apenas comparar os graus de escolarização nas quatro formas básicas de inferência. Verificou-se que os grupos com pouca ou nenhuma escolarização cometeram mais erros nas formas inválidas do que o de universitários. Após a coleta de dados, realizou-se conversas informais com os sujeitos de pouco escolarização. Alguns pediram explicações sobre as soluções dos problemas. Várias vezes foram oferecidos exemplos do tipo: "Olha, quando a gente diz 'Se a água está fervendo está quente’ não é a mesma coisa que dizer 'Se a água está quente está fervendo.” E, muitas vezes, os próprios sujeitos verbalizavam "É claro, às vezes está quente mas não chegou ainda à fervura e aí quando a senhora pergunta 'a água tá quente, ela tá fervendo', o certo era a gente dizer 'talvez', não é?’. Assim, sem falar sobre "recíproca", que parece não ser uma palavra usual entre sujeitos com pouca escolarização, mas "contrário", tentou-se, neste estudo, minimizar o grande número de erros encontrados em outros estudos.

\section{Método}

\section{Participantes}

Fizeram parte deste estudo 30 sujeitos sendo dez estudantes universitários, dez adultos analfabetos e dez
Tollens, Afirmação do Conseqüente e Negação do Antecedente). O conteúdo dos mesmos envolveram fatos que concordaram com a experiência diária dos sujeitos. Cada problema consistiu em duas premissas e uma conclusão em forma de pergunta (Ver Tabela 1 para exemplo). Três opções de respostas foram oferecidas "Pode ser ou pode não ser", "Sim" e "Não". As justificativas foram requeridas após as respostas dos sujeitos.

Cada sujeito foi entrevistado individualmente. Aos universitários (Grupo A) o experimentador (E) verbalizou que seriam apresentados alguns problemas e que eles deveriam responder "Sim", ou "Não" ou "Pode ser ou pode não ser/Talvez". Foi ainda acrescentado que eles deviam lembrar que nem toda "Recíproca é verdadeira" exemplificando "Se a água está fervendo está quente" não implica dizer que "Se a água está quente está fervendo". Aos adultos analfabetos (Grupo B) e aqueles com escolarização mínima (Grupo C), as instruções foram as mesmas apenas mudando a verbalização de que "Nem sempre a recíproca é verdadeira" para "Nem sempre o contrário é verdadeiro".

O E lia cada problema em voz alta e pedia ao sujeito para repeti-lo. Em caso de erros, o E lia novamente o problema. Após a resposta do sujeito, pedia-se que ele justificasse sua resposta. "Por que você disse "sim/não/ talvez?"

\section{Resultados}

Todas as respostas foram analisadas de dois modos: a) por resposta correta e b) por tipo de justificativa oferecido à resposta independentemente se a mesma era correta ou não.

Tabela 1. Exemplo de Problemas Silogísticos em Função da Forma

\begin{tabular}{llll}
\hline Modus Ponens & Modus Tollens & Afirmação do Conseqüente & Negação do Antecedente \\
\hline Se a luz está acesa então & Se a luz está acesa então & Se a luz está acesa então & Se a luz está acesa então tem \\
tem eletricidade. & tem eletricidade. & tem eletricidade. & eletricidade. \\
A luz da sala de Carlos & A sala de Carlos não tem & A sala de Carlos tem & A luz da sala de Carlos não \\
está acesa, & eletricidade, & eletricidade, & está acesa, \\
Tem eletricidade? & A luz está acesa? & A luz está acesa? & Tem eletricidade? \\
\hline
\end{tabular}

com escolaridade mínima (quarta série do Ensino Fundamental). Visto que, em nenhum estudo tem sido relatado diferenças entre sexos no desempenho em problemas silogísticos, esta variável não foi considerada.

\section{Material e Procedimentos}

Foram apresentados oito problemas silogísticos sendo dois de cada forma básica (Modus Ponens, Modus

\section{Respostas Corretas}

A Tabela 2 apresenta as médias de acertos e os desviospadrão nos quatro tipos de silogismo. Foi realizada uma Análise de Variância para amostras repetidas, tendo como fatores os três grupos e como variável dependente (VD) o número de respostas corretas nas quatro formas básicas de silogismo. Esta análise não mostrou efeito significativo para grupo, $F(2,27)=1,59 ; p=0,22$. 
394 Maria da Graca Bompastor Borges Dias

Tabela 2 . Médias (Desvios-Padrão) de Respostas Corretas Oferecidas em cada Grupo em cada Forma de Silogismo (máximo de 2)

\begin{tabular}{|c|c|c|c|}
\hline $\begin{array}{ll}\text { Frupos } & \text { Formas }\end{array}$ & MP & MT & NA \\
\hline GA & $1,50(0,53)$ & $0,60(0,84)$ & $1,10(0,57)$ \\
\hline GB & $1,90(0,32)$ & $1,80(0,63)$ & $0,30(0,67)$ \\
\hline GC & $1,90(0,32)$ & $1,90(0,32)$ & $0,50(0,85)$ \\
\hline TOTAL & $1,77(0,43)$ & $1,43(0,86)$ & $0,63(0,76)$ \\
\hline
\end{tabular}

Legenda: GA = Universitário; GB = Adultos analfabetos; GC = Adultos com escolarização mínima

No entanto, a análise estatística comparando as médias de acertos nos quatro tipos de silogismos mostrou efeito significativo para tipo de silogismo, $F(3,81)=23,87$ $p<0,001$, e uma interação entre grupos e tipo de silogismo, $F(6,81)=14,95 ; p<0,001$.

Comparando as médias em cada tipo de silogismo através do teste de Newman-Keuls, verifica-se que as médias de acertos nos silogismos Modus Ponens e Modus Tollens foram significativamente maiores $(p<0,01)$ do que as médias nos tipos Negação do Antecedente e Afirmação do Conseqüente. Todas as outras comparações não mostraram efeito significativo.

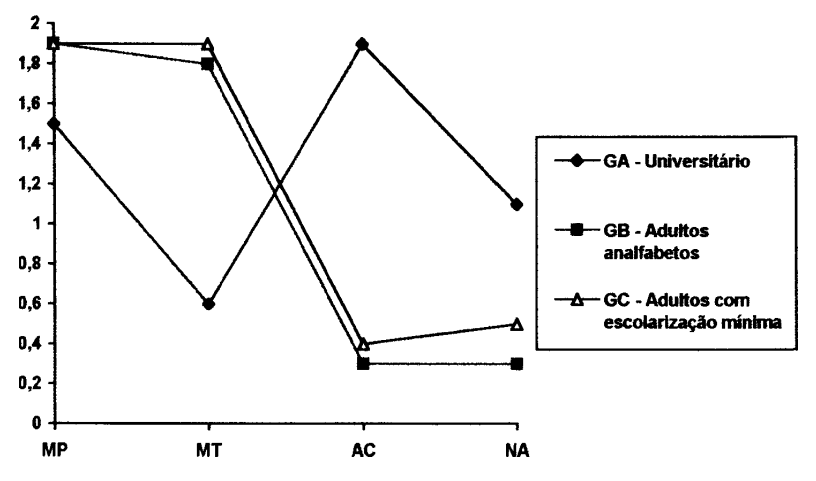

Figura 1. Interação entre grupos e tipos de silogismos
A interação entre grupo e tipo silogismo (ver Figura 1) e comparações específicas entre pares de médias envolvidas na interação entre grupo e tipo de silogismo foram realizadas através de contrastes. Entre os sujeitos de pouca ou nenhuma escolarização não houve diferenças significativas nas quatro formas de silogismos. No entanto o grupo de universitário obteve significativamente menor número de acertos $(p<0,01)$ do que os outros dois grupos na forma Modus Tollens. No entanto o desempenho dos universitários foi significativamente melhor $(p<0,01)$ nas formas inválidas do que os outros dois grupos. As outras comparações não mostraram diferenças significativas.

\section{Tipo de Justificativa}

As justificativas foram categorizadas como teóricas, empíricas ou arbitrárias, segundo os critérios utilizados por Dias e colaboradores (Dias, 1996; Dias \& Harris, 1988a, 1988b, 1990). Considerou-se justificativas teóricas aquelas em que o sujeito explicava sua resposta referindose às premissas dadas. As empíricas referiam-se à experiência do dia-a-dia do sujeito. E as arbitrárias foram justificativas irrelevantes ou quando o sujeito não justificava. As justificativas foram classificadas por dois juizes independentes e treinados. O grau de concordância entre juizes foi de $96,7 \%$ em 240 justificativas. As

Tabela 3. Média(Desvio- Padrão) de Justificativas Teóricas, Empíricas em cada Tipo de Silogismo em função do Grupo (máximo de 2)

\begin{tabular}{|c|c|c|c|c|c|c|c|c|c|c|c|c|c|c|c|}
\hline \multirow{2}{*}{ Grupo } & \multicolumn{4}{|c|}{ Teóricas } & \multirow[t]{2}{*}{ Total } & \multicolumn{4}{|c|}{ Empíricas } & \multirow[t]{2}{*}{ Total } & \multicolumn{4}{|c|}{ Arbitrárias } & \multirow[t]{2}{*}{ Total } \\
\hline & MP & MT & AC & NA & & MP & MT & AC & NA & & MP & MT & AC & NA & \\
\hline GA & $\begin{array}{c}1,50 \\
(0,71)\end{array}$ & $\begin{array}{c}0,20 \\
(0,63)\end{array}$ & $\begin{array}{c}0,30 \\
(0,68)\end{array}$ & $\begin{array}{c}0,20 \\
(0,63)\end{array}$ & $\begin{array}{c}2,20 \\
(2,20)\end{array}$ & $\begin{array}{c}0,50 \\
(0,71)\end{array}$ & $\begin{array}{c}1,80 \\
(0,63)\end{array}$ & $\begin{array}{c}1,60 \\
(0,70)\end{array}$ & $\begin{array}{c}1,80 \\
(0,63)\end{array}$ & $\begin{array}{c}5,70 \\
(2,20)\end{array}$ & - & - & $\begin{array}{c}0,10 \\
(0,32)\end{array}$ & - & $\begin{array}{c}0,10 \\
(0,32)\end{array}$ \\
\hline$g C$ & $\begin{array}{c}1,60 \\
(0,52)\end{array}$ & $\begin{array}{c}1,80 \\
(0,42)\end{array}$ & $\begin{array}{c}1,20 \\
(0,63)\end{array}$ & $\begin{array}{c}1,30 \\
(0,82)\end{array}$ & $\begin{array}{c}5,90 \\
(1,44)\end{array}$ & $\begin{array}{c}0,40 \\
(0,52)\end{array}$ & $\begin{array}{c}0,20 \\
(0,42)\end{array}$ & $\begin{array}{c}0,80 \\
(0,63)\end{array}$ & $\begin{array}{c}0,60 \\
(0,84)\end{array}$ & $\begin{array}{c}2,00 \\
(1,50)\end{array}$ & - & - & - & $\begin{array}{c}0,10 \\
(0,32)\end{array}$ & $\begin{array}{c}0,10 \\
(0,32)\end{array}$ \\
\hline & $\begin{array}{c}1,40 \\
(0,70)\end{array}$ & $\begin{array}{c}1,70 \\
(0,68)\end{array}$ & $\begin{array}{c}1,30 \\
(0,82)\end{array}$ & $\begin{array}{c}1,10 \\
(0,99)\end{array}$ & $\begin{array}{c}5,50 \\
(2,50)\end{array}$ & $\begin{array}{c}0,60 \\
(0,70)\end{array}$ & $\begin{array}{c}0,30 \\
(0,68)\end{array}$ & $\begin{array}{c}0,70 \\
(0,82)\end{array}$ & $\begin{array}{c}0,80 \\
(0,92)\end{array}$ & $\begin{array}{c}2,40 \\
(2,30)\end{array}$ & - & - & - & - & - \\
\hline
\end{tabular}

Legenda: $\mathrm{GA}=$ Universitário; $\mathrm{GB}=$ Adultos analfabetos; $\mathrm{GC}=$ Adultos com escolarização mínima 
classificações discrepantes (8) foram julgadas por um terceiro juiz, cujas classificações finais coincidiram com uma das duas realizadas anteriormente.

As médias dos tipos de justificativa oferecidas pelos grupos nas quatro formas de problemas silogísticos encontram-se na Tabela 3.

Verifica-se que o grupo de universitários utilizou menos justificativas Teóricas do que os outros dois grupos, cujas explicações deste tipo foram em grande número. Para checar esta diferença, foi realizada uma Anova tendo como fator os grupos e como variável dependente (VD) as justificativas teóricas. Esta análise mostrou um efeito significativo, $F(2,29)=9,52 ; p<0,001$. Análises posteriores com o T-teste denota que o GA recorreu menos às teóricas do que o GB $(p<0,001)$ e o GC $(p<0,005)$. Esses dois últimos grupos não diferiram.

As justificativas empíricas foram mais utilizadas pelo GA e muito menos pelos outros dois grupos. A ANOVA envolvendo grupos e justificativas empíricas mostrou um efeito significativo de Grupo, $F(2,29)=10,08 ; p<0,001$. A comparação das médias através do T-teste mostrou que o GA recorreu em maior número às justificativas empíricas do que o GB $(p<0,001)$ e do que o GC $(p<0,004)$. A comparação entre os dois últimos, não se mostrou significativa.

Por ter havido apenas duas justificativas arbitrárias, uma oferecida a um problema envolvendo Afirmacão do Conseqüente, e outra envolvendo Negação do Antecedente, não foram realizadas as análises estatísticas.

\section{Discussão}

Um dos resultados mais saliente deste estudo foi a boa performance nas formas válidas dos sujeitos com menos ou nenhuma escolarização quando comparados aos universitários. Em vários estudos citados anteriormente (Dias, 1987; Dias \& Harris, 1988a, 1988b, 1990; Shapiro \& O'Brien, 1970, entre outros), os dados denotam que tanto crianças como adultos obtêm grande número de acertos na forma válida, Modus Ponens, decaindo um pouco na forma Modus Tollens. Quanto as formas inválidas, as investigações mostram um desempenho muito baixo em diferentes grupos de sujeitos.

No presente estudo, apenas os dois grupos com baixa ou nenhuma escolarização comportaram-se de tal modo. Os universitários alcançaram um alto número de acertos nas formas inválidas, compatível com aquele que ocorria em outros estudos quando adultos e mesmo crianças se depararam com problemas envolvendo as formas válidas. No entanto, os universitários obtiveram baixo desempenho nessas formas válidas.

Psicologia: Reflexão e Crítica, 2000, 13(3), pp.391-397
Surpreendente, também, foram os tipos de justificativa utilizados pelos diferentes grupos. Os sujeitos com baixa ou nenhuma escolarização obtiveram desempenho similar, utilizando em maior escala justificativas teóricas e, em menor, justificativas empíricas. O contrário ocorreu com os universitários que recorreram em maior número às justificativas empíricas e, diferentemente dos resultados alcançados em outros estudos, suas explicações teóricas foram poucas.

Verifica-se, portanto, que a inclusão das variáveis "nem sempre a recíproca/o contrário é verdadeira(o)" juntamente com a alternativa "pode ser ou pode não ser" tiveram um efeito positivo no número de acertos dos sujeitos universitários nas formas inválidas, embora tenham tido um efeito negativo nas formas válidas. Os universitários, tendiam a responder "talvez" nos problemas envolvendo Modus Ponens e Modus Tollens, o que não acontece quando esta alternativa não é oferecida. Já os sujeitos de pouca ou nenhuma escolarização parecem não ter sido influenciados por esta alternativa. Permaneceram com bom desempenho nas formas válidas e baixo, nas inválidas, como mostra a literatura, isto é, respondendo sim para a forma Afirmação do Conseqüente e não para a forma Negação do Antecedente. No entanto, essas variáveis tiveram influência no tipo de justificativa que esses sujeitos de pouca ou nenhuma escolarização escolheram para suas respostas. Também diferentemente da literatura, a procura por justificativas teóricas foi grande e inesperada e as arbitrárias, encontradas em grande número em outros estudos, foram aqui mínimas.

Pode-se concluir que a tentativa de minimizar o número de respostas erradas nas formas inválidas, foi alcançada, pelo menos para os universitários. Mas quanto às suas justificativas e às suas respostas às formas válidas, que estiveram aquém daqueles de outros estudos? Será que o engajamento no sistema educacional (no caso a universidade) levaria os sujeitos a serem mais cuidadosos em suas opções, escolhendo aquela alternativa menos determinista? Outra questão que ainda se encontra em aberto é a de como melhorar o número de respostas corretas daqueles com pouca ou nenhuma escolarização nas formas inválidas, apesar de que a qualidade de suas justificativas, neste estudo, ter sido superior àquela encontrada em investigações anteriores.

No entanto, um possível esclarecimento para os dados aqui alcançados pode ser apoiada pela Teoria da Lógica Mental proposicional (Braine \& O’Brien, 1991; Braine, O'Brien, Noveck, Samuels, Lea, Fish \& Yang, 1995; O’Brien, 1993, 1995). Esta fornece uma explicação do tipo de inferências dedutivas que os sujeitos fazem a partir de partículas lógicas como se, e, não e ou. Assim, a lógica 
proposicional consiste em um conjunto de esquemas de inferências lógicas que dizem respeito à conjunção, disjunção, negação e condicionais, e um programa de raciocínio para a aplicação dos esquemas. Os estudos realizados por esses pesquisadores averiguaram as inferências dos sujeitos durante processamento de textos, discursos, como também quando os mesmos resolvem problemas silogísticos. A maioria desses estudos procurou evidências desses esquemas lógicos proposicionais em adultos (e.g., Lea, 1998; Lea, O’Brien, Fish, Noveck \& Braine, 1990). Entre crianças tem-se apoio ao modelo através dos trabalhos de Braine e Rumain (1981), O’Brien, Braine, Cornell, Noveck, Fish e Fun (1989), O’Brien, Dias Roazzi e Braine (1998), Bloom, Lahey, Hood, Lifter e Feiss (1980) e Bowerman (1986).

A teoria da lógica mental apresenta três partes que serão aqui discutidas brevemente. Uma parte consiste de um conjunto de esquemas de inferências como por exemplo, quando alguém sabe que duas proposições na forma $p$ ou $q$ e não $p$ são verdadeiros, $q$ pode ser concluído O conjunto de esquemas básicos que aqui importam são aqueles que estão incluídos nas inferências lógicas que os indivíduos fazem rotineiramente e não todos os tipos que eles seriam capazes de fazer.

A segunda parte da teoria consiste em um programa de raciocínio que aplica os esquemas em linhas de raciocínio e inclui uma rotina direta (raciocínio mais simples), e uma estratégia mais sofisticada (raciocínio indireto). A primeira linha seria universal e aplicável com esforço mínimo tanto no raciocínio como na compreensão. Segundo os defensores dessa teoria, podese predizer que as inferências que são elaboradas, aplicando-se os esquemas básicos (Modus Ponens e o Modus Tollens) com o raciocínio direto, são feitas rotineiramente. Esses são aplicados corretamente mesmo por crianças pré-escolares (ver O’Brien, Dias \& Braine, 1998). O que pode justificar o excelente desempenho do grupo com pouca ou nenhuma escolarização.

O’Brien, Dias, Roazzi e Braine (1998) argumentam ainda, que existem evidências para estratégias mais sofisticadas, aplicáveis nas inferências inválidas, entre universitários e presumivelmente, em outras populações, porém essas não seriam universais e seriam meno freqüentes que as estratégias básicas. "As estratégias de raciocínio indireto são capacidades secundárias adquiridas mais tarde, que são sujeitas à variação individual embora comum em sujeitos adultos" (Braine \& O’Brien, 1998, p. 79). Isto poderia explicar o baixo desempenho do grupo menos escolarizado ou analfabeto nas inferências inválidas.

A terceira parte da teoria é pragmática. A teoria propõe que o significado lógico básico de cada partícula encontra-se nas inferências que são sancionadas pelos esquemas de inferências. Os esquemas de inferências atuariam nas representações semânticas que seriam o output dos processos de compreensão. Logo, os fatores pragmáticos que influenciam a compreensão são afetadas pela informação através da qual as inferências são realizadas.

Há então três princípios gerais:

- A estratégia da plausibilidade da interpretação da sentença: os sujeitos interpretaram mais acuradamente uma proposição quando esta envolve seus conhecimentos específicos e gerais do mundo.

- O princípio da cooperatividade: o falante tenta ser verdadeiro o quanto possível, informativo, claro em suas exposições e, do mesmo modo, o ouvinte interpreta as exposições assumindo que as mesmas são verdadeiras, informativas, etc. Por este motivo, esse princípio oferece margem a muitas inferências desnecessárias (implicações conversacionais), o que pode ser uma fonte de erros em tarefas lógicas. Isto talvez tenha ocorrido com os sujeitos universitários do presente estudo nas inferências válidas.

- Provocação de inferências: Por exemplo, um condicional se p então $q$ provoca e inferência se não $p$ então não $q$; uma sentença com ou chamaria uma inferência com ambos não, e assim por diante (conversão inválida). Os sujeitos fazem erros deste tipo a não ser que haja algum contramando que mostraria ser desapropriado (ver Dias, 1996).

Este tipo de investigação não pode parar por aqui. Procurar-se-á, em próximos estudos, conseguir resultados satisfatórios para essas questões.

\section{Referências}

Bloom, L., Lahey, M., Hood, L., Lifter, K. \& Feiss, K. (1980). Complex sentences: Acquisition of syntactic connectives and the semantic relations they enconde. Journal of Child Language, 7, 235-261.

Bowerman, M. (1986). First steps in acquiring conditionals. Em E. Traugott, A. ter Meulen, J. S. Reilly \& C. A. Ferguson (Orgs.), On conditionals (pp. 285-307). Cambridge, England: Cambridge Univerty Press.

Braine, M. D. S. \& O'Brien, D. P. (1991). A theory of if: A lexical entry, reasoning program, and pragmatic principles. Psychological Review, 98, 182203

Braine, M. D. S. \& O’Brien, D. P. (1998). Mental logic. Mahwah, N.J: Lawrence Erlbaum.

Braine, M. D. S., O'Brien, D. P., Noveck, I. A., Samuels, M., Lea, R. B., Fisch, S. M. \& Yang, Y. (1995). Predicting intermediate and multiple conclusions in propositional logic inference problems: Further evidence for a mental logic (Número especial). Journal of Experimental Psychology, 94.

Braine, M. D. S. \& Rumain, B. (1981). Development of comprehension of "or": Evidence for a sequence of competencies. Journal of Experimental Cbild Psychology, 31, 46-70

Chapman, L. J. \& Chapman, J. P. (1959). Atmosphere effect re-examined. Journal of Experimental Psychology, 58, 220-226. 
Dias, M. G. B. B. (1987). Da lógica do analfabeto à lógica do adolescente: Há progresso? Arquivos Brasileiros de Psicologia, 39, 29-40.

Dias, M. G. B. B. (1996). O desenvolvimento do raciocínio dedutivo. E M. G. Dias \& A. G. Spinillo (Orgs.), Tópicos em Psicologia Cognitiva (pp. 11-44). Recife: Editora da UFPE - Série Estudos Universitários.

Dias, M. G. \& Harris, P. L. (1988a). The effect of make-believe play on dedutive reasoning. British Journal of Developmental Psychology, 6, 207-221.

Dias, M. G. B. B. \& Harris, P. L. (1988b). Realidade X fantasia: Sua influência no raciocínio dedutivo. Psicologia: Teoria e Pesquisa, 4, 55-68.

Dias, M. G. B. B. \& Harris, P. L. (1990). The influence of the imagination on reasoning by young children. British Journal of Developmental Psychology, 8, 305-318.

Dias, M. G. B. B. \& Ruiz, E. L. (1990). Bloqueando a conversão inválida. Arquivos Brasileiros de Psicologia, 42, 66-77.

Lea, R. B. (1998). Logical inferences and comprehension: How mentallogic and text processing theories need each other. Em M. D. S. Braine \& D. P. O'Brien (Orgs.), Mental logic (pp. 63-78). Mahwah, NJ Lawrence Erlbaum.

Lea, R. B., O'Brien, D. P., Fisch, S. M., Noveck, I. A. \& Braine, M. D. S. (1990). Predicting propositional logic inferences in text comprehesion. Journal of Memory and Language, 29, 361-387.

Falmagne, J. A. (1975). Reasoning: Representation and process in cbildren and adults. Hillsdale, NJ: Lawrence Erlbaum.

Mayer, R. E. (1977). Thinking and problem solving: An introduction to buman cognition and learning. Glenview, IL: Scott, Freeman \& Company.

O'Brien, D. P. (1993). Mental logic and irrationality: We can put a man on the moon, so why can't we solve those logical reasoning tasks? Em K. I. Manktelow \& D. E. Over (Orgs.), Rationality: Psychological and philosoppical perspectives (pp. 110-135). London: Routledge.

O'Brien, D. P. (1995). Finding logic in human reasoning requires looking in the right places. Em S. E. Newstead \& J. St. B. T. Evans (Orgs.), Perspectives on thinking and reasoning: Essays in honour of Peter Wason (pp. 189-216). Hove, UK: Lawrence Erlbaum.

O'Brien, D. P., Braine, M. D. S., Connell, J. W., Noveck, I. A., Fisch, S. M. \& Fun, E. (1989). Reasoning about conditional sentences: Development of understanding of cues to quantification. Journal of Experimental Child Psychology, 48, 90-113.
O’Brien, D. P., Dias, M. G., Roazzi, A. \& Braine, M. D. (1998). Conditional reasoning: The logic of supposition and children's understanding of pretence. Em M. D. S. Braine \& D.P. O’Brien (Orgs.), Mental logic (pp. 245-272). Mahwah, NJ: Lawrence Erlbaum.

Revlis, R. (1975a). Syllogistic reasoning: logical decisisions from a complex data base. Em R. Falmagne (Org.), Reasoning: Representation and process in cbildren and adults (pp. 93-133). Hillsdale, NJ: Lawrence Erlbaum.

Revlis, R. (1975b). Two models of syllogistic reasoning: Feature selection and conversion. Journal of Verbal Learning and Verbal Behavior, 14, 180195.

Rips, L. J. \& Marcus, S. L. (1977). Supposition and the analysis of conditional sentences. Em M. A. Just \& P. A. Carpenter (Orgs.), Cognitive processes in comprehension. Hillsdale, NJ: Lawrence Erlbaum.

Rumain, B., Connell, J. \& Braine, D. S. (1983). Conversational comprehension processes are responsible for reasoning fallacies in children as well as adults: If is not the biconditional. Developmental Psychology, 19, $471-481$.

Shapiro, B. J. \& O’Brien, T. C. (1970). Logical thinking in children ages six through thirteen. Child Development, 41, 823-829.

Taplin, J. E. \& Staudenmaver, H. (1973). Interpretation of abstract conditiohal sentences in deductive reasoning. Journal of Verbal Learning and Verbal Behavior, 12, 530-542

Wildman, T. M. \& Fletcher, H. J. (1977). Developmental increases and decreases in solutions of conditional syllogism problems. Developmental Psychology, 13, 630-636.

Sobre a autora

Maria da Graça Bompastor Borges Dias é Psicóloga, Doutora pela University of Oxford, Inglaterra,

Bolsista do CNPq, Professora da Graduação e Pós-Graduação em Psicologia da UFPE. 


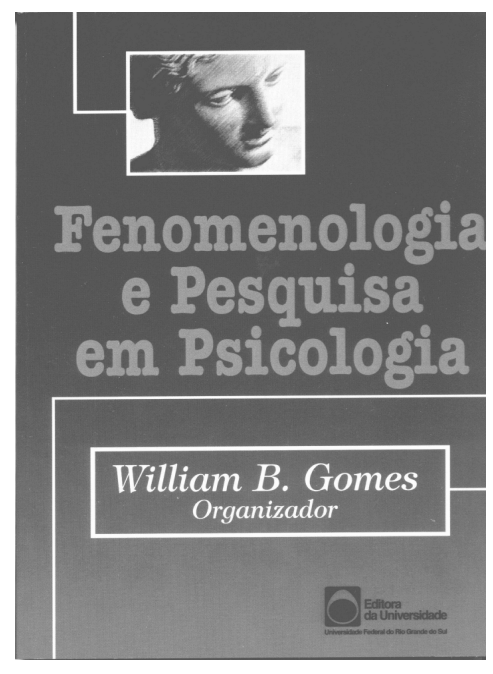

\section{Sumário}

1. Entrevista Fenomenológica e Pesquisa em Psicologia William B. Gomes

2. Concepções de Alcoolismo e a Reabilitação do Alcoolismo José Carlos Leite e W. B. Gomes

3. Adolescência e Expectativas em Relação aos Efeitos do Álcool Lisiane B. Araújo e W. B. Gomes

4. Adolescência jovens portadores de doenças orgânicas crônicas

Viviane Z. de Oliveira e W. B. Gomes

5. Relatos de Mães em Transformações Familiares em Três Gerações

Ciomara R. S. Benicá e William |B. Gomes

6. Escolha Vocacional em Adolescentes

Mauro Magalhães, M. Célia Lassance e William B. Gomes

7. Grupos Homogêneos com Obesos

W. B. Gomes, Marco Teixeira e Márcia Borges

Pedidos à Editora da Universidade. Av. João Pessoa, 415, 90040.000

Porto Alegre, RS. Fone/Fax: (51) 2248821, 3164082, 3164090

http://www.ufrgs.br/editora - E-mail: editora@ orion.ufrgs.br 